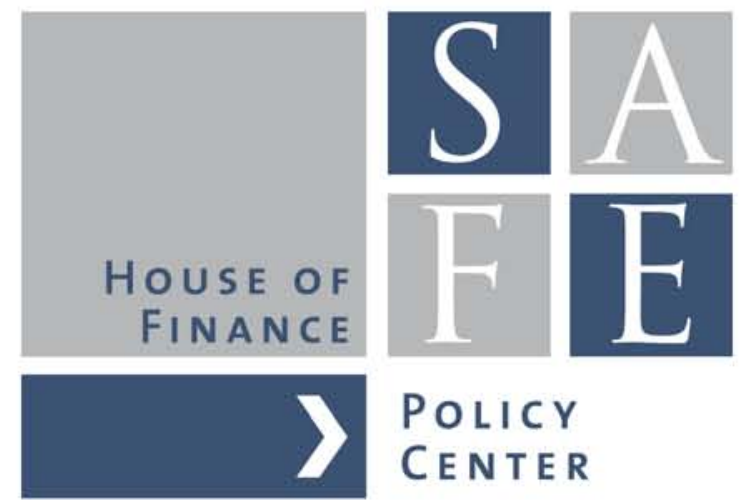

Peter Gomber und Frank Nassauer

\title{
Neuordnung der Finanzmärkte in Europa durch MiFID II/MiFIR
}

White Paper Series No. 20

SAFE I Sustainable Architecture for Finance in Europe

A cooperation of the Center for Financial Studies and Goethe University Frankfurt 
Dieses Papier ist erschienen in: Zeitschrift für Bankrecht und Bankwirtschaft, Volume 26, Issue 42014

SAFE Policy papers represent the authors' personal opinions and do not necessarily reflect the views of the Research Center SAFE or its staff. 


\title{
Neuordnung der Finanzmärkte in Europa durch MiFID II / MiFIR
}

\author{
Peter Gomber, Frank Nassauer ${ }^{*}$
}

Die Anpassung der EU-Richtlinie über Märkte für Finanzinstrumente (MiFID II) und die Einführung einer begleitenden Verordnung (MiFIR) im Jahr 2014 werden erhebliche Auswirkungen auf die Finanzmärkte in Europa haben und zu einer grundlegenden Neuordnung der Finanzmarktstrukturen führen. Ausgehend von einer Diskussion der Zielerreichung der ursprünglichen Richtlinie (MiFID I) aus dem Jahr 2004 werden im vorliegenden Artikel die Zielsetzungen und Maßnahmen der Neuregelung beleuchtet. Wesentliche Elemente im Hinblick auf Marktstrukturen und den Wertpapierhandel sind die Einführung einer neuen Handelsplatzkategorie, des organisierten Handelssystems („Organised Trading Facility"; OTF), sowie die Ausweitung der bislang für Aktien geltenden Transparenzvorschriften auf weitere Finanzinstrumente. Zudem werden eine Handelsverpflichtung für Aktien und Derivate sowie eine Clearingpflicht für Derivate, die auf geregelten Märkten gehandelt werden, neu eingeführt. Schließlich werden der algorithmische Handel und der Hochfrequenzhandel auf europäischer Ebene reguliert, wobei die Regelungen weitgehend dem 2013 eingeführten deutschen Hochfrequenzhandelsgesetz angelehnt sind. Im Ausblick wird zunächst der weitere Prozess der Regulierung skizziert (insbesondere die sog. Level II-Maßnahmen). Abschließend werden mögliche Auswirkungen von MiFID II und MiFIR auf die Marktstruktur und den Wertpapierhandel aufgezeigt.

\section{Inhaltsübersicht}

I. Einleitung

II. Entwicklung der Ziele und Maßnahmen der MiFID II / MiFIR auf Basis der MiFID I

III. Zentrale Inhalte der MiFID II / MiFIR in Bezug auf Marktstrukturen und Wertpapierhandel

1. Marktstruktur

2. Erweitertes Transparenzregime und Einschränkung von Transparenzausnahmen

3. Handelsverpflichtung für Aktien und Derivate

4. Clearingpflicht

5. Algorithmischer Handel / Hochfrequenzhandel

IV. Ausblick und Diskussion

\footnotetext{
* Prof. Dr. Peter Gomber leitet die Professur für Betriebswirtschaftslehre, insbesondere e-Finance der Goethe-Universität Frankfurt a.M., Dr. Frank Nassauer, Spezialist Unternehmensentwicklung im Vorstandsstab der Deutschen Apotheker- und Ärztebank, Düsseldorf.
} 


\section{Einleitung}

Europa hat ein neues Grundgesetz für den Wertpapierhandel und die Organisation der Marktstruktur. Basierend auf dem Vorschlag der Europäischen Kommission vom 20. Oktober $2011^{1}$ haben nach über zweijähriger Diskussion das Europäische Parlament am 15. April 2014 und der Europäische Rat am 13. Mai 2014 eine neue Richtlinie über Märkte für Finanzinstrumente in Europa (MiFID $\left.\mathrm{II}^{2}\right)$ und eine begleitende Verordnung $\left(\mathrm{MiFIR}^{3}\right)$ verabschiedet. Die neuen Regelwerke wurden am 12. Juni 2014 im Amtsblatt der Europäischen Union veröffentlicht und sind am 2. Juli 2014 in Kraft getreten. 24 Monate nach der Veröffentlichung und dem Inkrafttreten, d.h. am 3. Juli 2016, sollen diese in das jeweilige nationale Recht der Mitgliedsstaaten umgesetzt sein. Sechs Monate nach diesem Zeitpunkt, also 30 Monate nach dem Inkrafttreten ${ }^{4}$ und damit am 3. Januar 2017, sollen die neuen Regelwerke durch Wertpapierfirmen, Marktbetreiber, Datenbereitstellungsdienste und Drittlandfirmen, die Wertpapierdienstleistungen oder Anlagetätigkeiten in der EU erbringen ${ }^{5}$, angewendet werden.

Wesentliche Neuerungen der MiFID II und der MiFIR in Bezug auf den Wertpapierhandel und die Marktstruktur beziehen sich auf die Einführung einer neuen Form von Handelsplätzen, den sogenannten Organised Trading Facilities (OTF), ein erweitertes Transparenzregime (inklusive Bestrebungen zur Konsolidierung der Nachhandelsdaten) und die Einschränkung der bisherigen Transparenzausnahmen, eine Handelsverpflichtung für Aktien und Derivate, eine Clearingverpflichtung für börsengehandelte Derivate sowie neue Regelungen für den algorithmischen Handel bzw. den Hochfrequenzhandel. Darüber hinaus finden sich in dem neuen Regelwerk eine Vielzahl von Maßnahmen zur Verbesserung des Anlegerschutzes, zum Beispiel strengere Anforderungen an die Portfolioverwaltung, die Anlageberatung und das Angebot komplexer Finanzprodukte sowie Maßnahmen zur Vermeidung potenzieller Interessenkonflikte. Der Fokus dieser Arbeit liegt auf den Neuerungen für den Wertpapierhandel und die Marktstruktur und berücksichtigt nicht das separate Feld des Anlegerschutzes.

Im Folgenden werden zunächst die Ziele der MiFID II / MiFIR für den Bereich Handel und Marktstrukturen erarbeitet und dargestellt. Als Basis hierfür werden die wesentlichen Maßnahmen der MiFID I sowie deren Erfolg unter Verweis auf die entsprechende wissenschaftliche Literatur diskutiert. Daran anschließend werden die wesentlichen Neuerungen und Maßnahmen der Richtlinie und der Verordnung umfassend erläutert. Ein Ausblick auf den weiteren Umsetzungsprozess und eine Diskussion möglicher Auswirkungen des neuen Regelwerks auf die europäischen Finanzmärkte schließen die Arbeit ab.

\footnotetext{
Vgl. Europäische Kommission (2011).

Vgl. Europäisches Parlament und Rat (2014a) - nachfolgend referenziert als: (MiFID II).

Vgl. Europäisches Parlament und Rat (2014b) - nachfolgend referenziert als: (MiFIR).

Vgl. Art. 93 Abs. 1 der Richtlinie (MiFID II) sowie Art. 55 der Verordnung (MiFIR).

Vgl. Art. 1 Abs. 1 der Richtlinie (MiFID II).
} 


\section{Entwicklung der Ziele und Maßnahmen der MiFID II / MiFIR auf Basis der MiFID I}

Seit dem 1. November 2007 sind die Bestimmungen der "Markets in Financial Instruments Directive“ (MiFID) - diese ursprüngliche Richtlinie wird im Folgenden als MiFID I bezeichnet - entsprechend der nationalen Umsetzung von allen Unternehmen, die Wertpapierdienstleistungen erbringen, und den geregelten Märkten europaweit anzuwenden. Bei der Veröffentlichung des ersten Vorschlages ${ }^{6}$ für die MiFID I am 19. November 2002 hat die Europäische Kommission als zentrale Zielsetzung die Gewährleistung der Integrität und die Förderung transparenter, effizienter und integrierter Finanzmärkte angeführt. Weiterhin betont die Europäische Kommission die Notwendigkeit, „die europäischen Wertpapiermärkte wettbewerbsfähiger [zu] machen“ mit der Absicht, „Wertpapiere mit einem Maximum an Effizienz und einem Minimum an Kosten zu handeln“ ${ }^{7}$.

Die zentralen Neuerungen der MiFID ${ }^{8}$, die der Umsetzung dieser Ziele dienen sollen, sind die Neudefinition und Klassifikation von Handelsplätzen (Geregelte Märkte, Multilaterale Handelssysteme, Systematische Internalisierer), die Einführung von weitreichenden Transparenzvorschriften für den OTC-Handel (was insbesondere für Deutschland neu war), die Definition von „Best Execution“ auf europäischer Ebene, und veränderte Organisationspflichten und Wohlverhaltensregeln bei der Durchführung von Wertpapierdienstleistungen. Insbesondere die Einführung einheitlicher Wettbewerbsbedingungen zwischen Börsen und Wertpapierdienstleistungsunternehmen und die damit verbundene Entstehung neuer Handelsplätze, der sogenannten Multilateral Trading Facilities (MTF) ${ }^{9}$, hat die europäische Marktstruktur grundlegend verändert und zu einer erheblichen Fragmentierung geführt. Die MiFID I klassifiziert die verschiedenen Mechanismen der Orderausführung in geregelte Märkte und MTFs, die multilaterale Handelsplätze darstellen, sowie in Systematische Internalisierer $(\mathrm{SI})^{10}$ und Over-The-CounterGeschäfte (OTC), die als bilaterale Ausführungen gelten. Für diese Mechanismen wurden weitreichende Vor- und Nachhandelstransparenzanforderungen eingeführt. Geregelte Märkte und MTFs haben einheitliche Vorhandelstransparenzanforderungen ${ }^{11}$ für Aktien entsprechend dem jeweiligen Handelsmodell zu erbringen. Die Vorhandelstransparenz im Fall der Systematischen Internalisierung $^{12}$ ist durch das Stellen verbindlicher Kauf- und Verkaufsangebote für Aktiengattungen, für die ein liquider Markt besteht, zu erfüllen. Im OTC-Bereich besteht weiterhin keine Verpflichtung zur Vorhandelstransparenz ${ }^{13}$. Die Bestimmungen zur Nachhandelstransparenz gelten grundsätzlich gleichermaßen für die verschiedenen Handelsplätze ${ }^{14}$ einschließlich des OTC-

\footnotetext{
Vgl. Europäische Kommission (2002a), S. 9.

Vgl. Europäische Kommission (2002b).

Vgl. Europäisches Parlament und Rat (2004).

Vgl. Europäisches Parlament und Rat (2004), Art. 4 Abs. 1 (15).

Vgl. Europäisches Parlament und Rat (2004), Art. 4 Abs. 1 (7).

Vgl. Europäisches Parlament und Rat (2004), Art. 29 und Art. 44.

Vgl. Europäisches Parlament und Rat (2004), Art. 27.

Vgl. Europäisches Parlament und Rat (2004), Erwägungsgrund 53

Vgl. Europäisches Parlament und Rat (2004), Art. 30 und Art. 45.
} 
Geschäfts $^{15}$ und verpflichten dazu, Preis, Volumen und Zeitpunkt aller Aktiengeschäfte zu veröffentlichen. Grundidee der MiFID I ist es, die möglichen Nachteile der Fragmentierung für die Effizienz des Preisbildungsprozesses durch diese umfangreichen Transparenzvorschriften abzumildern.

Vor der Anwendbarkeit der MiFID I existierte in vielen europäischen Mitgliedsstaaten, zum Beispiel Frankreich, Italien oder Spanien, noch ein Börsenzwang und in Deutschland konnten aufgrund des Börsenvorranges Kundenorders nur bei ausdrücklicher Zustimmung des Kunden außerbörslich ausgeführt werden, was zu einer häufig kritisierten monopolartigen Stellung und zu Markanteilen der jeweiligen Heimatbörsen nahe $100 \%$ führte. Im Gegensatz dazu ist die europäische Wertpapierlandschaft heute stark fragmentiert und es ist den neuen MTF wie Chi-X, BATS ${ }^{16}$ oder Turquoise gelungen, den europäischen Börsen in den meisten Hauptaktienindizes Markanteile von über $20 \%{ }^{17}$ abzunehmen. Die mit der MiFID I eingeführte Kategorie der Systematischen Internalisierer, die insbesondere für den Handel von Orders zwischen Wertpapierunternehmen auf eigene Rechnung mit ihren direkten Kunden in Transaktionsgrößen unterhalb der Standardmarktgröße ausgelegt ist, hat mit nur zwölf Häusern, die Systematische Internalisierung betreiben ${ }^{18}$, und einem Marktanteil von zum Teil unter $3 \%{ }^{19}$ keine relevante Bedeutung erlangt.

Dieser neue Wettbewerb konnte sehr positive Wirkungen auf die expliziten und impliziten Transaktionskosten im europäischen Wertpapierhandel entfalten. Die niedrigeren Gebühren der neu in den Markt eingetretenen MTF haben bei den etablierten Börsen und auch im Bereich der Wertpapierabwicklung bei Zentralen Kontrahenten (CCP) und Wertpapiersammelbanken (CSD) zu deutlichen Gebührenreduktionen geführt. ${ }^{20}$ Eine Vielzahl akademischer Arbeiten haben darüber hinaus empirisch einen positiven Effekt des Wettbewerbs auf die Marktliquidität in Form reduzierter impliziter Transaktionskosten nachweisen können. ${ }^{21}$ Damit hat der europäische Gesetzgeber das Ziel der Kostenreduktion durch Wettbewerb klar erreicht.

Neben dieser neuen Wettbewerbslandschaft im Bereich transparenter Marktplätze existiert aber weiterhin ein stabiler und hoher Anteil des außerbörslichen Handels von etwa $40 \%{ }^{22}$, in dem eine Vielzahl von kleinen Transaktionen durchgeführt werden, die auf den transparenten Markplätzen keinen negativen Markteinfluss generieren würden. ${ }^{23}$ Auch hat das vermehrte Aufkommen nicht

\footnotetext{
15 Vgl. Europäisches Parlament und Rat (2004), Art. 28.

16 Die MTFs BATS Europe and Chi-X Europe haben in 2011 eine Fusion zu BATS Chi-X Europe vollzogen und im Mai 2013

die Zulassung als Geregelter Markt erhalten.

17 Vgl. Thomson Reuters (2014).

18 Vgl. European Securities and Markets Authority (2014a).

19 Vgl. Fidessa (2014).

20 Vgl. Oxera (2011).

21 Vgl. zum Beispiel Gomber et al. (2011 a), Gresse (2012), Hengelbrock et al. (2009), Soltani et al. (2011), oder Riordan et al. (2011).

22 Vgl. Thomson Reuters (2014).

23 Vgl. Gomber et al. (2011 b).
} 
transparenter Handelsplätze, sogenannter „Dark Pools“ ${ }^{24}$, sowohl im Bereich der geregelten Märkte und MTFs als auch im OTC-Bereich (dort oftmals als „Broker Crossing Networks" bezeichnet) zu vielfältiger Kritik ${ }^{25}$ wegen einer nicht einheitlichen Umsetzung der Transparenzvorschriften und zu breiten Transparenzausnahmen geführt, wobei hier oftmals die möglichen negativen Auswirkungen mangelnder Vorhandelstransparenz auf die Liquidität angeführt werden. ${ }^{26}$

Zur Sicherstellung des Investorenschutzes in einem fragmentierten Umfeld hat die MiFID I weitreichende Vorschriften für die Ausführung von Kundenorders formuliert. In deren Zentrum stehen die Verpflichtungen zur bestmöglichen Ausführung von Kundenorders ${ }^{27}$ („Best Execution“) für alle Finanzinstrumente sowohl in Bezug auf Privatkunden als auch auf professionelle Kunden. Wertpapierfirmen haben Ausführungsgrundsätze festzulegen („Best Execution Policy“) und dem Kunden offenzulegen, die mindestens jährlich überprüft werden müssen. Dabei sind alle relevanten Kriterien zur Erzielung des bestmöglichen Ergebnisses zu berücksichtigen und im Hinblick auf die Merkmale des Kunden, des Kundenauftrags, des jeweiligen Finanzinstruments und der infrage kommenden Ausführungsplätze zu gewichten. Das Best Execution-Konzept der MiFID I wird von vielen Marktbeobachtern als nicht erfolgreich gewertet. Die Grundsätze der Auftragsausführung sind oftmals wenig konkret und werden von den meisten Häusern nicht als Wettbewerbsinstrument und Basis für eine Bank- bzw. Brokerauswahl sondern als regulatorische Pflicht gesehen. ${ }^{28}$

Die Europäische Kommission hebt in ihrer eigenen Bewertung ${ }^{29}$ der MiFID I den europaweiten Wettbewerb zwischen Börsen und alternativen Handelsplätzen sowie die damit verbundene Reduzierung der Transaktionskosten sowie eine erhöhte Integration der Märkte hervor. Auch sieht sie die erweiterten Möglichkeiten für die europaweite Erbringung von Dienstleistungen für Wertpapierfirmen und das breitere Spektrum an Dienstleistungen für den Anleger als positiv an. Jedoch werden auch die aufgetretenen Probleme nach Einführung der MiFID I aufgelistet: (i) die Tatsache, dass die Vorteile des Wettbewerbs nicht immer an die Endinvestoren weitergegeben wurden, und die mit der Fragmentierung verbundene erhöhte Komplexität, (ii) das Auftreten neuer Markt- und Technologieentwicklungen, die bei der Diskussion zur MiFID I noch nicht vorhersehbar waren, (iii) die erkennbaren Schwächen der Regulierung im Kontext der Finanzkrise und (iv) die schnelle Innovation und hohe Komplexität bei Finanzinstrumenten.

\footnotetext{
24 Als Dark Pool wird eine bank- oder börseninterne Handelsplattform für den anonymen Handel von Finanzprodukten bezeichnet. Das zentrale Merkmal von Dark Pools ist ihre fehlende (Vorhandels-)Transparenz, d.h. es wird nicht öffentlich angezeigt, wie viele Wertpapiere zu welchen Kursen angeboten oder nachgefragt werden.

25 Vgl. Puaar (2012).

26 Vgl. Degryse et al. (2011).

27 Vgl. Europäisches Parlament und Rat (2004), Art. 21.

28 Zur Bewertung des Best Execution-Konzeptes in der akademischen Literatur vgl. Gomber et al. (2012), Ferrarini (2007) oder Petrella (2010)

29 Vgl. Europäische Kommission (2011).
} 
Aus dieser Bestandsaufnahme werden die wesentlichen Ziele ${ }^{30}$ für die Überarbeitung der MiFID I abgeleitet $^{31}$ : (i) die Positionierung der MiFID II als integraler Bestandteil eines neuen Finanzsystems nach der Krise, (ii) die weitere Umsetzung der G20-Beschlüsse von Pittsburgh und der Folgetreffen in Bezug auf die Regulierung von OTC-Derivaten, (iii) die Verbesserung der Überwachung und Transparenz im Bereich der Warenderivate, (iv) die Sicherstellung eines fairen Wettbewerbs und effizienter Märkte vor dem Hintergrund der veränderten Marktstrukturen und der technologischen Entwicklungen und (v) die Reduktion der Ermessensspielräume für Mitgliedsstaaten durch ein einheitliches Regelwerk. Der letztgenannte Punkt ist der wesentliche Treiber für die Aufspaltung der MiFID-Überarbeitung in zwei neue Gesetzesvorhaben: eine Verordnung („,Markets in Financial Instruments Regulation“, MiFIR), die primär die Markttransparenz, die Handelsverpflichtungen, die Meldeverpflichtungen und den diskriminierungsfreien Zugang zu Clearingeinrichtungen abdeckt und nach Inkrafttreten unmittelbar in allen Mitgliedsstaaten ohne nationale Umsetzung gültig ist, und eine Richtlinie („Markets in Financial Instruments Directive“, MiFID II), die unter anderem die Anforderungen an Handelsplätze und Datendienstleister, die Organisationspflichten und Wohlverhaltensregeln für Wertpapierfirmen und die Rechte der zuständigen Behörden regelt.

\section{Zentrale Inhalte der MiFID II / MiFIR in Bezug auf Marktstrukturen und Wertpapierhandel}

\section{Marktstruktur}

Mit der Neujustierung der Richtlinie (MIFID II) und der Einführung einer Verordnung (MiFIR) über Märkte für Finanzinstrumente soll sichergestellt werden, dass der Handel in Finanzinstrumenten so weit wie möglich an organisierten Handelsplätzen stattfindet und diese Handelsplätze angemessen reguliert sind. Dabei erkennen das Europäische Parlament und der Rat an, dass sich in den vergangenen Jahren Handelssysteme entwickelt haben - genannt werden explizit Broker Crossing Networks - die von den Regelungen der Richtlinie zuvor nicht hinreichend erfasst wurden. ${ }^{32}$

Die Vorschriften zu geregelten Märkten und Multilateralen Handelssystemen (MTF) werden klarer gefasst. So wird den Betreibern eines MTF untersagt, Kundenaufträge gegen das eigene Buch auszuführen oder sich in der Zusammenführung sich deckender Kundenaufträge („Matched Principal Trading“) zu betätigen. ${ }^{33}$ Weitaus bedeutsamer ist jedoch, dass eine dritte Kategorie für den multilateralen Handel eingeführt wird: das Organisierte Handelssystem (OTF). Begründet wird die

30 Vgl. Europäische Kommission (2011).

31 Darüber hinaus hat gemäß Art. 63 der MiFID I die Erstellung von Berichten und die Überprüfung von Regulierungsinhalten der MiFID I, zum Beispiel eine Ausweitung der Transparenzvorschriften auf weitere Assetklassen neben Aktien oder die Vorschriften zu Systematischen Internalisierung zu erfolgen.

32 Vgl. Erwägungsgrund (6) der Verordnung (MiFIR).

33 Vgl. Art. 19 Abs. 5 der Richtlinie (MiFID II). Beim Matched Principal Trading tritt ein Mittler zwischen Käufer und Verkäufer ein, ohne das Marktrisiko während der Ausführung der Transaktion zu übernehmen. Beide Seiten werden simultan und zu einem Kurs ausgeführt, bei dem der Mittler keinen Gewinn oder Verlust erzielt, abgesehen von der zuvor veröffentlichten Provision oder Gebühr für die Transaktion. Vgl. Art. 4 Abs. 1 (38) der Richtlinie (MiFID II). 
Einführung dieser neuen Kategorie damit, die europäischen Märkte transparenter und effizienter zu machen und zudem gleiche Wettbewerbsbedingungen („Level Playing Field“) für die verschiedenen Märkte, die multilateralen Handel anbieten, zu gewährleisten. ${ }^{34}$

Ein Organisiertes Handelssystem (OTF) ist definiert als ein multilaterales System, das weder ein geregelter Markt noch ein MTF ist und das die Interessen einer Vielzahl Dritter am Kauf und Verkauf von Schuldverschreibungen, strukturierten Finanzprodukten, Emissionszertifikaten oder Derivaten innerhalb des Systems in einer Weise zusammenführt, die zu einem Vertrag gemäß den Bestimmungen des Titel II der Richtlinie führt. ${ }^{35}$ D.h. für den Aktienhandel ist ein OTF nicht zulässig. ${ }^{36}$ Die umfassenden Transparenzanforderungen, die Verpflichtung zur bestmöglichen Ausführung für den Kunden („Best Execution“) sowie die organisatorischen Anforderungen gelten auch für OTFs. Wie ein MTF kann auch ein OTF von einer Wertpapierfirma oder einem Marktbetreiber betrieben werden. Um die Neutralität des Betreibers zu wahren, ist eine Ausführung von Kundenaufträgen gegen das eigene Buch des OTF-Betreibers oder eines gruppenangehörigen Unternehmens nicht erlaubt. Matched Principal Trading erfordert die Zustimmung des Kunden; es ist gänzlich untersagt in Derivaten, die der Clearingpflicht unterliegen. Bei öffentlichen Schuldtiteln ohne einen liquiden Markt ist dagegen ein Handel gegen das eigene Buch sowie Matched Principal Trading erlaubt. Im Falle der Anwendung von Matched Principal Trading gelten allerdings die Regelungen zur Vor- und Nachhandelstransparenz sowie zur kundengünstigsten Ausführung („Best Execution“). Ferner ist das Betreiben eines OTF und eines Systematischen Internalisierers innerhalb desselben Unternehmens unzulässig. ${ }^{37}$

Abgesehen davon, dass ein OTF für den Aktienhandel nicht zur Verfügung steht, besteht der wesentliche Unterschied zu geregelten Märkten und MTFs darin, dass der Betreiber (Träger) eines OTF bei der Zusammenführung von Aufträgen einen Ermessensspielraum im Hinblick auf Interaktionen von Aufträgen im System hat (diskretionärer Handel). ${ }^{38}$ Die Wertpapierfirma bzw. der Marktbetreiber kann diesen Ermessensspielraum in zweifacher Weise ausüben: Zum einen bei der Entscheidung über die Einstellung in oder die Rücknahme eines Auftrags aus dem Handelssystem. Zum zweiten bei der Entscheidung über die Zusammenführung eines Auftrags mit den im System verfügbaren Aufträgen zu einem bestimmten Zeitpunkt. Grundsätzlich besteht allerdings ein Anspruch auf diskriminierungsfreie Ausführung und diskriminierungsfreien Zugang. Daher muss den Kunden vorab dargelegt werden, wie ein mögliches Ermessen ausgeübt wird. Der Träger des OTF darf

\footnotetext{
34 Vgl. Erwägungsgrund (8) der Verordnung (MiFIR).

35 Vgl. Art. 4 Abs. 1 (23) der Richtlinie (MiFID II).

36 Ein OTF darf auch nicht für den Handel von Aktienzertifikaten („Depositary Receipts“), börsengehandelten Fonds („,Exchange-traded Funds“), Zertifikaten und anderen aktienähnlichen Finanzinstrumenten genutzt werden.

37 Vgl. Art. 20 Abs. 1-4 der Richtlinie (MiFID II).

38 Bei einem geregelten Markt oder einem MTF hat die Zusammenführung der Handelsinteressen innerhalb des Systems und nach nicht-diskretionären Regeln zu erfolgen. Es besteht also für den Betreiber des jeweiligen Marktes kein Ermessensspielraum bei der Ausführung von Aufträgen; maßgebend sind die Regeln des Systems, das Systemprotokoll oder seine internen Ausführungsprozesse.
} 
in keinem Fall gegen Kundenanweisungen verstoßen und muss auch beim Ausüben des Ermessens die Verpflichtung zur kundengünstigsten Ausführung erfüllen. ${ }^{39}$

Nach Auffassung des Europäischen Parlaments und des Rates ist die Definition der neuen Handelskategorie breit genug, um künftig alle Typen der organisierten Ausführung von Kundenaufträgen und Vermittlung von Transaktionen abzudecken, die bislang nicht den regulatorischen Spezifikationen der bestehenden Handelsplatzkategorien entsprachen. ${ }^{40}$

Im Gegensatz zum multilateralen Handel bleibt die Klassifikation im Bereich des bilateralen Handels unverändert. Der bilaterale Handel erfolgt entweder als Systematischer Internalisierer oder ist ansonsten ein Over-the-Counter-Geschäft (OTC). Im Hinblick auf die Definition des Systematischen Internalisierers wurde jedoch eine Nachjustierung vorgenommen. Ein Systematischer Internalisierer ist demnach eine Wertpapierfirma, die in organisierter und systematischer Weise häufig und in erheblichem Umfang für eigene Rechnung handelt, in dem sie Kundenaufträge außerhalb eines geregelten Marktes, eines MTFs oder eines OTFs ausführt, ohne ein multilaterales System zu betreiben. Dabei bemessen sich die Häufigkeit sowie die systematische Tätigkeit anhand der Zahl der OTC-Transaktionen mit einem Finanzinstrument, die die Wertpapierfirma für eigene Rechnung bei der Ausführung von Kundenaufträgen tätigt. Der erhebliche Umfang bemisst sich zum einen anhand des Anteils des OTC-Handels einer Wertpapierfirma an ihrer gesamten Handelsaktivität in einem bestimmten Finanzinstrument und zum anderen anhand des Anteils des OTC-Handels dieser Wertpapierfirma am Gesamthandel des betreffenden Finanzinstruments in der Europäischen Union. Nur wenn beide Schwellenwerte überschritten werden, liegt ein Systematischer Internalisierer vor. ${ }^{41}$

Wertpapierfirmen sind verpflichtet, für Aktien und aktienähnliche Finanzinstrumente, die auf einem Handelsplatz gehandelt werden, wo sie als Systematischer Internalisierer fungieren und für die ein liquider Markt vorliegt, verbindliche Kursofferten zu stellen. Sie können das Volumen festlegen, für die sie Kursofferten stellen. Allerdings schreibt die Verordnung nunmehr eine Mindestquotierungsgröße („Minimum Quote Size“) in Höhe von $10 \%$ der Standardmarktgröße vor. Systematische Internalisierer müssen Kursofferten regelmäßig und kontinuierlich während der Handelszeit publik machen. Sie können ihre Kursofferten jederzeit anpassen, eine Rücknahme von verbindlichen Kursofferten ist aber nur bei außergewöhnlichen Marktbedingungen zulässig. Systematische Internalisierer können bestimmen, welchen Kunden sie Zugang zu ihren Kursofferten gewähren, jedoch muss dieser dann für die betreffenden Kunden diskriminierungsfrei erfolgen. Diese Regeln zur Verpflichtung der Stellung von Kursofferten, zur Veröffentlichung dieser Kursofferten, zur Ausführung von Kundenaufträgen und zum Zugang zu Kursofferten gelten allerdings nicht, wenn

\footnotetext{
39 Vgl. Art. 20 Abs. 6 der Richtlinie (MiFID II).

40 Vgl. Erwägungsgrund (8) der Verordnung (MiFIR).

41 Vgl. Art. 4 Abs. 1 (20) der Richtlinie (MiFID II). Die Spezifizierung der Schwellenwerte obliegt der Europäischen Kommission mittels delegierter Rechtsakte. Vgl. Art. 2 Abs. 2 i.V.m. Art. 50 MiFIR.
} 
Systematische Internalisierer in Losgrößen handeln, die über der standardmäßigen Marktgröße liegen. ${ }^{42}$

\section{Erweitertes Transparenzregime und Einschränkung von Transparenzausnahmen}

Die Verbesserung der Transparenz ist eine der grundlegenden Maßnahmen zur Stärkung der Finanzsysteme, auf die sich die G20-Staaten in ihrer Erklärung vom 2. April 2009 verständigt haben. Um die Transparenz zu erhöhen und die Funktionsweise der Märkte für Finanzinstrumente im europäischen Binnenmarkt zu verbessern, wird das neue Rahmenwerk für die Transparenz von Transaktionen in Finanzinstrumenten innerhalb der Verordnung (MiFIR) geregelt, um europaweit einheitliche Regeln zu gewährleisten. ${ }^{43}$

Zudem werden die Transparenzanforderungen, die bislang nur für Aktien gelten, die an einem geregelten Markt zugelassen sind, auf weitere Finanzinstrumente ausgeweitet. Die umfassenden Regelungen zur Vor- und Nachhandelstransparenz gelten künftig also auch für den Handel mit Aktienzertifikaten („Depositary Receipts“), börsengehandelten Fonds, Zertifikaten und anderen aktienähnlichen Finanzinstrumenten sowie den Handel mit Schuldverschreibungen, strukturierten Finanzprodukten, Emissionszertifikaten und Derivaten. ${ }^{44}$ Ausnahmen von der Vorhandelstransparenz und eine Verzögerung bei der Nachhandelstransparenz sind nur in bestimmten definierten Fällen möglich.

Im Rahmen der Verordnung wurden diese Ausnahmetatbestände („Waiver Regime“) für die Herstellung von Vorhandelstransparenz bei Aktien angepasst und teilweise beschränkt. Demnach sind Ausnahmen künftig in folgenden Fällen zulässig: (1) Für Handelssysteme, die auf einem Referenzpreis basieren („Reference Price Waiver“), (2) für Handelssysteme, die ausgehandelte Geschäfte formalisieren („Negotiated Transaction Waiver“), (3) für Aufträge mit großem Volumen im Vergleich zum marktüblichen Geschäftsumfang („Large-in-scale Waiver“) sowie (4) für Auftragsverwaltungssysteme eines Handelsplatzes („Order Management Facility Waiver"). ${ }^{45}$ Diese Ausnahmetatbestände waren auch in der ursprünglichen Richtlinie bereits vorgesehen. Um negative Auswirkungen auf den Preisbildungsmechanismus an organisierten Märkten zu vermeiden, ist in der Verordnung aber eine neue Bestimmung enthalten, die die Nutzung des Reference Price Waivers und des Negotiated Transaction Waivers volumenmäßig beschränkt („Volume Cap Mechanism“). Demnach dürfen nur noch maximal $4 \%$ des Handels eines Finanzinstruments an einem Handelsplatz und höchstens $8 \%$ des Handels in diesem Finanzinstrument an allen Handelsplätzen in der

\footnotetext{
42 Vgl. Art. 14 Abs. 1-3, Art. 15 Abs. 1 sowie Art. 17 Abs. 1 der Verordnung (MiFIR).

43 Vgl. Erwägungsgrund (1) der Verordnung (MiFIR).

44 Vgl. Art. 3 und Art. 8 der Verordnung (MiFIR).

45 Vgl. Art. 4 Abs. 1 der Verordnung (MiFIR). Diese Ausnahmentatbestände gelten auch für aktienähnliche Finanzinstrumente.
} 
Europäischen Union unter diese beiden Ausnahmetatbestände für die Herstellung von Vorhandelstransparenz fallen. ${ }^{46}$ Diese volumenmäßige Beschränkung könnte sich insbesondere auf die künftige Bedeutung von Dark Pools innerhalb der Europäischen Union auswirken.

Durch die Verordnung werden die Transparenzvorschriften auch auf Schuldverschreibungen, strukturierte Finanzprodukte, Emissionszertifikate und Derivate erweitert. Auch für diese Finanzinstrumente wurden Ausnahmetatbestände für die Herstellung von Vorhandelstransparenz eingeführt: (1) Für große Orders in Relation zur Standardmarktgröße („Large-in-scale Waiver“), (2) für Auftragsverwaltungssysteme eines Handelsplatzes („Order Management Facility Waiver“), (3) für verbindliche Interessebekundungen in Preisanfragesystemen („Request-for-Quote Systems“) und sprachbasierten Handelssystemen („Voice Trading Systems“), die über einer spezifischen Größe für das Finanzinstrument sind und die Bereitsteller von Liquidität übermäßigen Risiken aussetzen würden, sowie (4) für Derivate, die nicht der Handelspflicht unterliegen, und andere Finanzinstrumente, für die es keinen liquiden Markt gibt. ${ }^{47}$

Um eine einheitliche Anwendung der Ausnahmetatbestände von der Vorhandelstransparenz für bestimmte Marktmodelle sowie Ordertypen und Ordergrößen zu gewährleisten, soll die Europäische Wertpapieraufsichtsbehörde (European Securities and Markets Authority, ESMA) bei der Prüfung und Genehmigung von Anträgen zur Ausnahme von der Vorhandelstransparenz sicher stellen, dass sie in Einklang mit den Vorschriften der Verordnung und künftiger delegierter Rechtsakte stehen. ${ }^{48}$

Im Bereich der Nachhandelstransparenz ist beim Handel von Aktien und aktienähnlichen Finanzinstrumenten eine verzögerte Veröffentlichung von Einzelheiten von Transaktionen gemäß ihrem Typ und ihrer Größe erlaubt. Dies gilt insbesondere für Transaktionen, die groß in Relation zur Standardmarktgröße sind (,„Large-in-scale Transactions“). ${ }^{49}$ Für den Handel in Nichteigenkapitalinstrumenten, also Schuldverschreibungen, strukturierten Finanzprodukten, Emissionszertifikaten oder Derivaten, gilt dies gleichermaßen. Zudem ist eine verzögerte Veröffentlichung auch bei Instrumenten ohne liquiden Markt zulässig sowie für Transaktionen, die Bereitsteller von Liquidität übermäßigen Risiken aussetzen würden. ${ }^{50}$

Der Wettbewerb von Handelsplätzen um die Ausführung von Kundenorders hat auch dazu geführt, dass relevante Handelsdaten nicht mehr zentral an einem Platz zur Verfügung stehen. Um die Nachteile dieser Fragmentierung zu adressieren, enthalten die Richtlinie (MiFID II) und die Verordnung (MiFIR) auch neue Regelungen zur Datenkonsolidierung. Insbesondere werden

\footnotetext{
46 Vgl. Art. 5 Abs. 1 der Verordnung (MiFIR). Diese volumenmäßige Beschränkung gilt jedoch nicht für ausgehandelte Geschäfte („Negotiated Transactions“) in Aktien sowie aktienähnlichen Finanzinstrumenten, für die kein liquider Markt besteht.

47 Vgl. Art. 9 Abs. 1 der Verordnung (MiFIR).

48 Vgl. Art. 4 Abs. 4, Art. 9 Abs. 2 sowie Erwägungsgrund (13) der Verordnung (MiFIR).

49 Vgl. Art. 7 der Verordnung (MiFIR).

$50 \mathrm{Vgl}$. Art. 11 der Verordnung (MiFIR).
} 
Institutionen eingeführt und geregelt, die eine solche Konsolidierung der Handelsdaten bewirken sollen. Hier sind zuerst sog. genehmigte Veröffentlichungssysteme („Approved Publication Arrangements"; APA) zu nennen, die Handelsdaten im Namen von Wertpapierfirmen veröffentlichen. Sie sollen die Nachhandelstransparenz des OTC-Handels verbessern und zur Konsolidierung von OTCDaten mit Daten von organisierten Handelsplätzen beitragen. ${ }^{51}$ Um dies zu ermöglichen, müssen Wertpapierfirmen einschließlich Systematische Internalisierer das Handelsvolumen und den Kurs sowie den Zeitpunkt des Abschlusses von OTC-Transaktionen in Finanzinstrumenten unter Nutzung von APAs veröffentlichen. ${ }^{52}$ Bedeutsam für die Datenkonsolidierung sind vor allem aber die Bereitsteller konsolidierter Datenträger („Consolidated Tape Provider“; CTP), die zur Einholung von Transaktionsdaten über Finanzinstrumente bei geregelten Märkten, MTFs, OTFs und APAs berechtigt sind und sie in einem kontinuierlichen elektronischen Live-Datenstrom konsolidieren, über den Kursund Handelsvolumendaten pro Finanzinstrument abrufbar sind..$^{53}$ Der europäische Regulator setzt bei der Konsolidierung der Handelsdaten somit auf eine marktwirtschaftliche Lösung, bei der verschiedene autorisierte Anbieter auf Basis von vordefinierten und überwachten Parametern im Wettbewerb miteinander stehen und bedarfsgerechte, innovative Lösungen entwickeln sollen.

\section{Handelsverpflichtung für Aktien und Derivate}

Um zu gewährleisten, dass mehr Handel auf organisierten Handelsplätzen oder durch Systematische Internalisierer stattfindet, werden Wertpapierfirmen verpflichtet, für den Handel mit Aktien einen geregelten Markt, ein MTF oder einen Systematischen Internalisierer bzw. einen vergleichbaren Drittstaatenmarkt zu nutzen. Erinnert sei hier daran, dass die neue Handelsplatzkategorie OTF für den Aktienhandel nicht zur Verfügung steht. Erfasst von der Handelspflicht sind Aktien, sofern sie an einem geregelten Markt zum Handel zugelassen sind oder an einem anderen Handelsplatz gehandelt werden. Die Verpflichtung gilt auch für den Eigenhandel von Wertpapierfirmen. Ausnahmen gibt es lediglich für nicht-systematisch, ad-hoc, unregelmäßig und nicht häufig stattfindende Transaktionen sowie Transaktionen zwischen geeigneten und/oder professionellen Gegenparteien, die nicht zur Preisfindung beitragen. ${ }^{54}$ Diese Ausnahmen dürfen nicht dazu genutzt werden, die neu eingeführten Beschränkungen zur Anwendung des Reference Price Waiver sowie des Negiotated Price Waiver zu umgehen oder um Broker Crossing Networks oder andere Crossing-Systeme zu betreiben. ${ }^{55}$

Eine Wertpapierfirma, die ein internes Handelssystem für die Ausführung von Kundenaufträgen in Aktien, Aktienzertifikaten, börsengehandelten Fonds, Zertifikaten oder anderen aktienähnlichen

\footnotetext{
51 Vgl. Art. 4 Abs. 1 (52) der Richtlinie (MiFID II).

52 Vgl. Art. 20 und 21 der Verordnung (MiFIR).

53 Vgl. Art. 4 Abs. 1 (53) der Richtlinie (MiFID II).

54 Vgl. Art. 23 Abs. 1 der Verordnung (MiFIR). Die ESMA soll technische Standards entwerfen, die spezifizieren, wenn eine Transaktion nicht zum Preisfindungsprozess beiträgt.

55 Vgl. Erwägungsgrund (11) der Verordnung (MiFIR).
} 
Finanzinstrumenten auf multilateraler Basis betreibt, muss als MTF zugelassen sein und alle relevanten Anforderungen erfüllen. ${ }^{56}$

Die Verpflichtung zum Handel auf organisierten Märkten gilt auch für Derivate. Finanzielle Gegenparteien (gemäß Art. 2 Abs. 8 EMIR) und große nicht-finanzielle Gegenparteien (oberhalb der Clearingschwelle gemäß Art. 10 Abs. 1b EMIR) sind demnach verpflichtet, alle (OTC-) Derivate, die der Clearingpflicht gemäß EMIR unterliegen und die hinreichend liquide sind, auf geregelten Märkten, MTFs, OTFs oder vergleichbaren Drittstaatenmärkten zu handeln. Diese Handelspflicht gilt nicht für gruppeninterne Transaktionen sowie Transaktionen gemäß den Übergangsbestimmungen des Art. 89 EMIR. $^{57}$ Sie gilt aber sehr wohl für den Handel in erfassten Derivate(klassen) zwischen oben genannten Gegenparteien bzw. Finanzinstituten und Gegenparteien aus Drittstaaten, sofern letztere der Clearingpflicht unterliegen würden, falls sie in der Europäischen Union ansässig wären. Sie gilt ferner auch für den erfassten Derivatehandel zwischen Finanzinstituten und anderen Gegenparteien aus Drittstaaten, sofern dadurch eine direkte, substanzielle und vorhersehbare Auswirkung innerhalb der Europäischen Union ausgeht. ${ }^{58}$

Die Festlegung der von der Handelspflicht erfassten Derivate(klassen) obliegt der Europäischen Kommission mittels technischer Regulierungsstandards auf Vorschlag der ESMA. ${ }^{59}$ Ferner soll die ESMA regelmäßig die Aktivitäten in Derivaten überwachen, die nicht der Handelspflicht an organisierten Märkten unterliegen, um einerseits Derivateklassen zu identifizieren, die systemische Risiken verursachen, und um andererseits eine regulatorische Arbitrage zwischen Derivatetransaktionen, die der Handelspflicht unterliegen, und solchen, die von der Handelspflicht befreit sind, zu verhindern. ${ }^{60}$

Nach Vorstellung des Europäischen Parlaments und des Rats soll die Einführung der Handelspflicht einen effizienten Wettbewerb zwischen verschiedenen Handelsplätzen ermöglichen. Daher sollen Handelsplätze keine exklusiven Rechte für den Handel von Derivaten, die der Handelspflicht auf organisierten Märkten unterliegen, beanspruchen und andere Handelsplätze nicht vom Handel in diesen Derivaten abhalten können. ${ }^{61}$ Entsprechend regelt die Verordnung, dass Derivate, die der Handelspflicht unterliegen, auf nicht-exklusiver und nicht-diskriminierender Basis für den Handel an einem geregelten Markt zugelassen oder an jedem anderen organisierten Handelsplatz gehandelt werden können. ${ }^{62}$ Um einen effektiven Wettbewerb im Derivatehandel zu gewährleisten, sehen es

\footnotetext{
Vgl. Art. 23 Abs. 2 der Verordnung (MiFIR).

Vgl. Art. 28 Abs. 1 der Verordnung (MiFIR).

Vgl. Art. 28 Abs. 2 der Verordnung (MiFIR).

Vgl. Art. 32 Abs. 1 (a) der Verordnung (MiFIR).

Vgl. Art. 28 Abs. 2 der Verordnung (MiFIR).

Vgl. Erwägungsgrund (28) der Verordnung (MiFIR).

Vgl. Art. 28 Abs. 3 der Verordnung (MiFIR).
} 
das Europäische Parlament und der Rat zudem als essenziell an, dass Handelsplätze einen diskriminierungsfreien und transparenten Zugang zu Zentralen Kontrahenten (CCPs) erhalten. ${ }^{63}$

\section{Clearingpflicht}

Durch EMIR wurde eine Clearingpflicht für standardisierte OTC-Derivate eingeführt. Börsengehandelte Derivate sind durch EMIR jedoch nicht erfasst. Um diese rechtliche Lücke zu schließen und Ungleichbehandlungen von OTC- und börsengehandelten Derivaten zu beseitigen, wird durch die Verordnung eine Clearingverpflichtung auch für Derivate eingeführt, die auf einem geregelten Markt gehandelt werden. D.h. der Betreiber eines geregelten Marktes muss sicherstellen, dass alle Derivatetransaktionen, die auf diesem Markt ausgeführt werden, durch eine Zentrale Gegenpartei (CCP) abgewickelt werden. Zudem sind CCPs, Handelsplätze und Wertpapierfirmen, die als Clearing-Mitglied agieren, verpflichtet, Derivatetransaktionen schnellstmöglich dem Clearing zuzuführen; dafür sind automatisierte Systeme zu nutzen. Erfasst sind hiervon alle börsengehandelten Derivate sowie Derivate, die der Clearingpflicht gemäß Art. 4 EMIR unterliegen, ferner auch Derivate, für die die beteiligten Parteien ein Clearing vereinbart haben. ${ }^{64}$

Durch die Verordnung wird zudem das Recht auf diskriminierungsfreien Zugang von Handelsplätzen an Zentrale Gegenparteien (CCPs) sowie von CCPs an Handelsplätze festgeschrieben, soweit dies nicht bereits durch EMIR für OTC-Derivate geregelt ist. ${ }^{65}$ Die Regelungen zum diskriminierungsfreien Zugang von Handelsplätzen und CCPs im Rahmen der Verordnung beziehen sich auf alle Finanzinstrumente, die auf einem geregelten Markt oder einem vergleichbaren Drittstaatenmarkt gehandelt werden. CCPs müssen Handelsplätzen Zugang zu ihrem Clearing auf transparenter und nicht-diskriminierender Basis gewähren. Dies bedeutet unter anderem, dass Handelsplätze keine Benachteiligung im Hinblick auf Sicherheitenanforderungen und Clearinggebühren erfahren dürfen. Das Gleichbehandlungsgebot gilt auch für das Netting von ökonomisch vergleichbaren Kontrakten sowie das Cross-Margining mit korrelierten Kontrakten, die vom selben CCP innerhalb eines Risikomodells abgewickelt werden, das den Anforderungen der EMIR entspricht. Ein CCP kann allerdings verlangen, dass ein Handelsplatz die operationellen und technischen Anforderungen einschließlich der Anforderungen an das Risikomanagement des betreffenden CCPs erfüllt. ${ }^{66}$ Reziprok wird das Recht der diskriminierungsfreien Anbindung von CCPs an Handelsplätze verankert. Ein Handelsplatz wird verpflichtet, einem CCP, das auf diesem Handelsplatz erfüllte Transaktionen in Finanzinstrumenten abwickeln möchte, auf transparenter und nicht-diskriminierender Basis Zugang

\footnotetext{
63 Vgl. Erwägungsgrund (28) der Verordnung (MiFIR).

$64 \mathrm{Vgl}$. Art. 29 der Verordnung (MiFIR).

65 Kleinere Handelsplätze und Zentrale Kontrahenten, die eng aneinander gebunden sind und nicht über die erforderliche technologische Kapazität verfügen, um mit den größeren Infrastrukturanbietern im Nachhandelsbereich zu konkurrieren, haben eine Opt-out-Option von den Regelungen zum diskriminierungsfreien Zugang zwischen Handelsplätzen und CCPs, die 30 Monate gilt und ggf. erneuert werden kann. Vgl. Art. 35 Abs. 1 i.V.m. Art. 36 Abs. 5 sowie Art. 36 Abs. 1 i.V.m. Art. 35 Abs. 5 der Verordnung (MiFIR).

${ }^{66}$ Vgl. Art. 35 Abs. 1 der Verordnung (MiFIR).
} 
zu seinen Kurs- und Datenströmen („Trade Feeds“) zu gewähren. Ausgenommen sind auch hier Transaktionen in OTC-Derivaten, um eine Überschneidung mit EMIR zu vermeiden. ${ }^{67}$ Handelsplätze dürfen CCPs den Zugang nur verwehren, wenn diese bestimmte Kriterien, die in technischen Regulierungsstandards definiert werden, nicht erfüllen. ${ }^{68}$

Zudem muss der Zugang eines Handelsplatzes an ein CCP - und umgekehrt - von den zuständigen Aufsichtsbehörden genehmigt werden. Diese Genehmigung setzt voraus, dass für den Zugang keine Interoperabilitätsvereinbarung für börsengehandelte Derivate erforderlich ist. Die Genehmigung ist außerdem zu untersagen, wenn die Funktionsweise der Märkte gefährdet würde, insbesondere durch eine Fragmentierung der Liquidität, oder wenn sich daraus systemische Risiken ergeben könnten. ${ }^{69}$

Bezüglich börsengehandelter Derivate soll die Europäische Kommission die Risiken für die Stabilität und Funktionsweise der Finanzmärkte der Europäischen Union, die sich aus den Bestimmungen über den offenen Zugang von Handelsplätzen und CCPs ergeben könnten, analysieren und hierüber dem Europäischen Parlament und dem Rat Bericht erstatten. Je nach Ergebnis der Analyse kann die Europäische Kommission die Anwendung der Art. 35 und 36 auf börsengehandelte Derivate für bis zu 30 Monate ab dem 3. Januar 2017 aussetzen. ${ }^{70}$

Damit das Recht auf Zugang zwischen Handelsplätzen und CCPs nicht ins Leere läuft, weil bestehende Lizenzen an Referenzwerten („Benchmarks“) als Basis für Finanzinstrumente dies verhindern, sehen das Europäische Parlament und der Rat es zudem als erforderlich an, Handelsplätzen und CCPs Zugang zu Lizenzen an Benchmarks sowie Angaben zur Zusammensetzung, zur Methode und zur Kursbildung von Benchmarks in Bezug auf Finanzinstrumente zu gewähren. Dieser Zugang hat auf einer fairen, angemessenen und diskriminierungsfreien Basis zu erfolgen. Zudem müssen die Konditionen der Lizenzvergabe angemessen sein. ${ }^{71}$ Diese Bestimmung wird allerdings erst 54 Monate nach Inkrafttreten der Verordnung, d.h. am 3. Januar 2019 wirksam. ${ }^{72}$ Für neue Benchmarks, die nach Inkrafttreten der Verordnung entwickelt werden, gilt generell eine Nichtanwendungsfrist der Pflicht zur Lizenzierung von 30 Monaten beginnend mit dem Zeitpunkt, an dem der Handel eines Finanzinstruments, das auf diese Benchmark referenziert, aufgenommen wurde bzw. an dem das betreffende Finanzinstrument zum Handel zugelassen wurde. ${ }^{73}$

\footnotetext{
67 Vgl. Art. 36 Abs. 1 der Verordnung (MiFIR).

68 Vgl. Art. 36 Abs. 3 i.V.m. Art. 36 Abs. 6 (a) der Verordnung (MiFIR). Diese technischen Regulierungsstandards sollen von der ESMA entworfen werden.

69 Vgl. Art. 35 Abs. 4 und Art. 36 Abs. 4 der Verordnung (MiFIR). Allerdings kann die Genehmigung erteilt werden, wenn der Handelsplatz und alle beteiligten CCPs mit einer Interoperabilitätsvereinbarung einverstanden sind und die daraus resultierenden Risiken für das etablierte CCP des Handelsplatzes durch eine dritte Partei abgesichert werden.

70 Vgl. Art. 52 Abs. 12 der Verordnung (MiFIR).

71 Vgl. Art. 37 Abs. 1 der Verordnung (MiFIR).

72 Vgl. Art. 55 der Verordnung (MiFIR).

73 Vgl. Art. 37 Abs. 2 der Verordnung (MiFIR).
} 


\section{Algorithmischer Handel / Hochfrequenzhandel}

In Deutschland wird der algorithmische Handel und der Hochfrequenzhandel bereits durch das Deutsche Hochfrequenzhandelsgesetz reguliert, das am 15. Mai 2013 in Kraft getreten ist ${ }^{74}$. Im Rahmen der Überarbeitung der Richtlinie über Märkte für Finanzinstrumente wird der Hochfrequenzhandel nun auch auf europäischer Ebene geregelt. Das Europäische Parlament und der Rat erkennen an, dass sich die Handelstechnologien im letzten Jahrzehnt signifikant weiterentwickelt haben und Marktteilnehmer Handelssysteme nutzen, bei denen ein Computeralgorithmus wesentliche Parameter einer Order bestimmt. ${ }^{75} \mathrm{Um}$ zu vermeiden, dass der algorithmische Handel zu Marktstörungen führt, werden umfangreiche Vorschriften eingeführt.

Gemäß der Neufassung der Richtlinie ist der Hochfrequenzhandel als Form des algorithmischen Handels definiert, der sich durch folgende Merkmale auszeichnet: ${ }^{76}$

a) Eine Infrastruktur, die auf eine Minimierung der Latenz abzielt und sich dabei Co-Location, Proximity Hosting und/oder direktem elektronischen Zugang mit Hochgeschwindigkeit bedient;

b) eine systemseitige Initiierung, Generierung, Weiterleitung („Routing“) und Ausführung von einzelnen Transaktionen oder Aufträgen ohne menschliches Zutun; sowie

c) eine hohe Anzahl von Ereignissen innerhalb eines Tages, die Aufträge, Kursofferten oder Löschungen begründen.

Analog zum deutschen Hochfrequenzhandelsgesetz wird auch auf europäischer Ebene eine Erlaubnispflicht für Hochfrequenzhändler eingeführt. Wertpapierfirmen, die algorithmischen Handel betreiben, müssen über effektive Systeme und Risikokontrollen verfügen, um zu gewährleisten, dass ihre Handelssysteme belastbar sind, keine fehlerhaften Aufträge generieren oder auf sonstige Weise die Funktionsfähigkeit eines Marktes stören. Sie müssen Vorkehrungen für den Ausfall ihrer algorithmischen Handelssysteme treffen, diese vollumfänglich testen und sorgsam überwachen. Zudem sind sie verpflichtet, sowohl der Aufsichtsbehörde ihres Heimatlandes als auch der Aufsichtsbehörde des Handelsplatzes anzuzeigen, dass sie algorithmischen Handel betreiben. Der Aufsichtsbehörde des Heimatlandes werden umfangreiche Auskunftsrechte eingeräumt. Sie kann insbesondere von der Wertpapierfirma verlangen, dass sie regelmäßig oder ad-hoc ihre algorithmischen Handelsstrategien, Einzelheiten zu den Handelsparametern sowie ihre wesentlichen Compliance- und Risikokontrollmaßnahmen darlegt. Eine Wertpapierfirma, die Hochfrequenzhandel betreibt, hat ferner umfassende Aufzeichnungspflichten. Sie muss alle platzierten Aufträge einschließlich Löschungen von Aufträgen, ausgeführten Aufträgen und Quotierungen speichern und der Aufsichtsbehörde auf Verlangen zur Verfügung stellen. ${ }^{77}$

\footnotetext{
Vgl. Bundesgesetzblatt (2013).

Vgl. Erwägungsgrund (59) der Richtlinie (MiFID II).

Vgl. Art. 4 Abs. 1 Nr. (40) der Richtlinie (MiFID II).

Vgl. Art. 17 Abs. 1 und 2 der Richtlinie (MiFID II).
} 
Besondere Pflichten bestehen für algorithmische Händler, die als Market Maker fungieren. Sie müssen während eines bestimmten Zeitraums während der Handelszeiten eines Handelsplatzes kontinuierlich für Liquidität sorgen, es sei denn, dass außergewöhnliche Umstände vorliegen. Die genauen Spezifikationen müssen von der Wertpapierfirma und dem jeweiligen Handelsplatz in einer schriftlichen Vereinbarung festgehalten werden. ${ }^{78}$

Geregelte Märkte werden verpflichtet, effektive Systeme und Prozesse einzurichten und Vorkehrungen zu treffen, um eine Störung des Handels durch algorithmische Handelssysteme zu vermeiden bzw. die im Falle von Störungen Gegenmaßnahmen vorsehen. Dazu gehören insbesondere eine Begrenzung des Verhältnisses von unausgeführten Aufträgen zu Transaktionen („Order-to-trade Ratio“) eines Handelsteilnehmers, die Möglichkeit zur Einschränkung des Handels sofern die Kapazitätsgrenze des Handelssystems erreicht wird sowie die Festlegung von Mindestgrößen für Tick Sizes. ${ }^{79}$ Handelsplätze sollen in der Lage sein, von algorithmischen Systemen generierte Aufträge also solche zu identifizieren, in dem sie von den Handelsteilnehmern eine entsprechende Kennzeichnung („Flagging“) verlangen, und die hinter solchen Aufträgen stehenden Personen identifizieren können. ${ }^{80} \mathrm{Um}$ faire Handelsbedingungen für alle Nutzer zu gewährleisten, werden Handelsplätze verpflichtet, ihre Co-Location-Services ${ }^{81}$ auf einer diskriminierungsfreien, fairen und transparenten Basis anzubieten. ${ }^{82}$ Geregelten Märkten ist es aber erlaubt, von Marktteilnehmern, die einen hohen Anteil von Auftragslöschungen im Vergleich zu ausgeführten Aufträgen aufweisen („Order-to-trade Ratio“), höhere Gebühren zu erheben. Höhere Gebühren können auch für exzessive Systemnutzung verlangt werden, d.h. von Marktteilnehmern, die Hochfrequenzhandel betreiben und dadurch die Kapazität des Handelssystems belasten. ${ }^{83}$

Nicht eingeführt wurde dagegen eine Mindesthaltefrist für Aufträge. Der Vorschlag im Entwurf des Europäischen Parlaments, eine solche Mindesthaltedauer von Aufträgen von 500 Millisekunden einzuführen, wurde aufgrund der befürchteten negativen Konsequenzen für die Marktliquidität und des Anreizes für Hochfrequenzhändler, diese gebundenen Aufträge bei neuen Nachrichten auszunutzen, deutlich kritisiert und letztlich verworfen.

\footnotetext{
78 Vgl. Art. 17 Abs. 3 der Richtlinie (MiFID II).

Vgl. Art. 48 Abs. 6 der Richtlinie (MiFID II).

Vgl. Art. 48 Abs. 10 der Richtlinie (MiFID II).

81 Allgemein versteht man unter Co-Location die Unterbringung und den Betrieb eigener Hosts in einem (fremden) Rechenzentrum. Betreiber von Handelsplattformen, wie Wertpapierfirmen oder Börsenorganisationen, bieten ihren Kunden solche Co-Location-Lösungen an. Dabei befinden sich die Handelssysteme der Teilnehmer im gleichen Rechenzentrum wie die Systeme des Betreibers der Handelsplattform, also in unmittelbarer physischer Nähe. Dies ist insbesondere für algorithmische Händler bzw. Hochfrequenzhändler interessant, da so noch geringere Latenzzeiten erzielt werden können.

82 Vgl. Art. 48 Abs. 8 der Richtlinie (MiFID II).

83 Vgl. Art. 48 Abs. 9 der Richtlinie (MiFID II).
} 


\section{Ausblick und Diskussion}

Für die Richtlinie und die Verordnung über Märkte für Finanzinstrumente sind jeweils in über zwanzig Bestimmungen delegierte Rechtsakte vorgesehen, deren konkrete Ausgestaltung der ESMA und der Europäischen Kommission obliegen (Level II-Maßnahmen). Die Richtlinie bzw. die Verordnung beinhalten mehr als 100 Anforderungen, zu denen ESMA regulatorische bzw. technische Regulierungsstandards zu definieren hat bzw. die Kommission bei der Formulierung delegierter Rechtsakte unterstützen soll. Die ESMA hat hierfür bereits im August 2012 neun Arbeitsgruppen eingerichtet, davon vier unter dem Standing Committee Secondary Markets. Mit der Veröffentlichung eines ersten Diskussions ${ }^{84}$ bzw. Konsultationspapiers ${ }^{85}$ hat die ESMA am 22. Mai 2014 die öffentliche Diskussion zu den Level II-Maßnahmen gestartet und um Feedback bis zum 1. August 2014 gebeten.

Im Sekundärmarktbereich liegt der Fokus der Level II-Arbeiten auf den Transparenzvorschriften sowie der Handelspflicht, dem Hochfrequenzhandel, den Zugangsrechten zu einem CCP, zu einem Handelsplatz und zu Benchmarks sowie organisatorischen Anforderungen an Handelsplätze. Erfahrungsgemäß könnten weitere Diskussions- und Konsultationspapiere folgen, um der ESMA eine Basis für die Erfüllung ihrer gestellten Aufgaben zu geben. Level II wird final nach der Transposition der Richtlinie in nationales Recht im zweiten Quartal 2016 erwartet und damit kann die Richtlinie zu Beginn 2017 Anwendung finden ${ }^{86}$.

Die neuen Regelungen der MiFID II und der MiFIR werden die europäischen Finanzmärkte grundlegend verändern und neu ordnen. Die europäischen Aktienmärkte werden insbesondere durch die Einführung einer Handelsverpflichtung für Aktien und damit verbunden einer höheren Bedeutung der Kategorie der Systematischen Internalisierer, die Veränderungen im Bereich der Dark Pools durch Volumenobergrenzen und die neuen Anforderungen für den algorithmischen Handel und den Hochfrequenzhandel betroffen. Im Bereich der Nichteigenkapitalmärkte, also für Schuldverschreibungen, strukturierte Finanzprodukte, Emissionszertifikate und Derivate, ist der größte Einfluss auf die Marktstrukturen und den Wettbewerb insbesondere durch die Ausdehnung der Transparenzvorschriften, die bisher lediglich für Aktien gelten, auf diese Assetklassen, durch die Einführung einer Handels- bzw. Clearingverpflichtung für Derivate und durch die neue HandelsplatzKategorie OTF zu erwarten.

\footnotetext{
84 Vgl. European Securities and Markets Authority (2014b).

$85 \mathrm{Vgl}$. European Securities and Markets Authority (2014c).

86 Analog zur ursprünglichen Richtlinie sieht auch die angepasste Richtlinie vor, dass die Europäische Kommission innerhalb von 56 Monaten nach dem Inkrafttreten der Richtlinie, d.h. vor dem 3. März 2019, nach Rücksprache mit ESMA einen Bericht zu ausgewählten Themen, u.a. zur Funktionsweise der Regulierung von OTFs, vorlegen und gegebenenfalls eine Überarbeitung der Richtlinie vorschlagen soll („Review Clause“). Vgl. Art. 90 Abs. 1 der Richtlinie (MiFID II).
} 
Die Handelsverpflichtung für Aktien wird Umfang und Struktur des heutigen OTC-Handels verändern und zu einer Verlagerung vieler heutiger OTC-Geschäfte in den Bereich der Systematischen Internalisierung führen und diese Kategorie, die bislang nur vergleichsweise wenig genutzt ${ }^{87}$ wird, deutlich aufwerten. Broker Crossing Networks können in ihrer heutigen Form zukünftig im Aktienhandel nicht mehr existieren; diese Plattformen müssen in das regulatorische Format eines MTF oder eines Systematischen Internalisierers gebracht werden. Hier wird sich zeigen, welche Kreativität in den Häusern entfaltet wird, um dieses erfolgreiche Geschäftsmodell trotz der neuen regulatorischen Vorgaben zu erhalten. Die Broker Crossing Networks sind ja selbst ein Resultat der Nutzung von Schlupflöchern aus der MiFID I.

Dark Pool Trading hat in den letzten Jahren deutlich an Bedeutung gewonnen und den Regulierer unter anderem veranlasst, Volumenobergrenzen zu definieren. Hier wird es sehr spannend sein, welche Reaktionen diese Schwellenwerte auf das Handelsverhalten und die Geschäftsmodelle von Dark Pools haben. Die Schwellenwerte werden zu strategischem Verhalten führen, indem Marktteilnehmer und Dark Pools durch spezifische Handelsstrategien und Ordertypen die Überschreitung der Schwellenwerte verhindern werden oder sogar Anreize erhalten dafür zu sorgen, dass Konkurrenten die Schwellenwerte überschreiten.

Dem Hochfrequenzhandel werden nun, nach der Einführung des deutschen Hochfrequenzhandelsgesetzes, auch europaweit deutlich höhere regulatorische Kosten auferlegt. Hier sind insbesondere die Zulassungspflichten für die entsprechenden Häuser und die Kennzeichnungspflicht von Algorithmen zu nennen. Welche Auswirkungen die Market MakingVerpflichtungen auf die Attraktivität des Hochfrequenzhandels haben, wird wesentlich von der konkreten Ausgestaltung der Level II-Vorgaben abhängen. Es ist ohne Zweifel aus deutscher Sicht sehr positiv zu sehen, dass der europäische Regulator das deutsche Hochfrequenzhandelsgesetz in vielen Teilen in der MiFID II reflektiert hat und sich damit die Umsetzungserfordernisse für deutsche Wertpapierdienstleister und Marktplätze in überschaubarem Rahmen halten werden.

Grundlegende Änderungen wird es auch bei den europäischen Finanzmärkten für andere Assetklassen als Aktien geben. Dies ergibt sich aus dem Zusammenspiel der Ausweitung der Transparenzvorschriften auf Schuldverschreibungen, strukturierte Finanzprodukte, Emissionszertifikate und Derivate, der Handels- und Clearingpflicht sowie der Einführung einer neuen Handelsplatzkategorie (OTF) für diese Assetklassen. Die Vorschriften der MiFID I haben zu einer deutlichen Verbesserung der Transparenz der Aktienmärkte in Europa geführt. Dies gilt insbesondere auch für den OTC-Markt. Eine Ausweitung der Transparenzvorschriften - bei gleichzeitiger Beschränkung der Ausnahmen von der Vor- und Nachhandelstransparenz - auf andere Finanzinstrumente ist daher konsequent und wird insbesondere die Transparenz des Handels von Anleihen und Derivaten, der derzeit weit überwiegend OTC stattfindet, beträchtlich erhöhen.

87 Vgl. European Securities and Markets Authority (2014a). 
Die Handelsverpflichtung und die Clearingpflicht werden gravierende Folgen für den Derivatehandel haben. Künftig müssen alle OTC-Derivate, die der Clearingpflicht gemäß EMIR unterliegen und für die eine ausreichende Liquidität besteht, auf geregelten Märkten, MTFs oder OTFs gehandelt werden. Dadurch wird sich der Anteil des OTC-Handels deutlich reduzieren; entsprechend erhöht sich der Anteil der regulierten, überwachten und transparenten Handelsplätze. Zur Erfüllung der Handelspflicht kann die Einführung des Organisierten Handelssystems (OTF) eine sinnvolle Lösung sein, um den Handel von standardisierten OTC-Derivaten auf organisierte Märkte zu überführen. Diese neue Handelsplatzkategorie ist einerseits deutlich strenger reguliert als der OTC-Handel, eröffnet andererseits aber einem Marktbetreiber einen gewissen Ermessensspielraum für die Ausführung von Kundenorders. Diese größere Flexibilität und schwächere Regulierung im Vergleich zu einem geregelten Markt und einem MTF könnte allerdings auch dazu führen, dass Marktbetreiber ihre Systeme für den Handel von Nichteigenkapitalinstrumenten in der Regel als OTF zulassen und insbesondere MTFs hier keine große Rolle spielen werden.

Einer der besonders strittigen Punkte der MiFID-Novelle in den Trilog-Verhandlungen zwischen Europäischem Parlament, Rat und der Kommission war der diskriminierungsfreie Zugang zu Handelsplätzen, Zentralen Gegenparteien (CCPs) und Benchmarks, vor allem für Derivate. Die Intention dieser Regelungen ist klar: Analog zu Aktien sollen auch für börsengehandelte Derivate die Voraussetzungen für einen Wettbewerb verschiedener Handelsplätze und Clearinganbieter unter fairen Bedingungen (,Level Playing Field“) geschaffen werden. Dabei ist allerdings zu beachten, dass Aktien- und Derivatemärkte erhebliche Unterschiede aufweisen. Beim Aktienmarkt erfolgt der Wettbewerb im Wesentlichen über die Gebühren und die Leistungsfähigkeit des Handelssystems (sowie ggf. weitere Parameter wie Ordertypen). Bei den Derivatemärkten kommt dagegen dem Risikomanagement eine herausragende Bedeutung zu. Dies ist nicht zuletzt eine Erkenntnis der Finanzkrise. Folglich sind mögliche positive Wirkungen eines stärkeren Wettbewerbs gegen eine Erhöhung der systemischen Risiken beim Derivatehandel abzuwägen. Dementsprechend wird die Europäische Kommission beauftragt, zunächst die Risiken für die Stabilität und Funktionsweise der europäischen Finanzmärkte zu analysieren, die sich aus einem offenen Zugang zu Handelsplätzen und Clearinganbietern bei börsengehandelten Derivaten ergeben können. Allerdings sieht die Verordnung abhängig vom Ergebnis dieser Analyse lediglich eine maximal 30-monatige Aussetzung des Inkrafttretens der betreffenden Regelungen vor, jedoch offenbar keine gänzliche Streichung. Folglich ist davon auszugehen, dass der diskriminierungsfreie Zugang zu Handelsplätzen und Clearinganbietern auch bei börsengehandelten Derivaten über kurz oder lang eingeführt wird - und dies dürfte weit reichende Folgen für die Finanzmärkte in Europa haben. Die derzeitigen Marktstrukturen bei börsengehandelten Derivaten, die durch eine enge Verzahnung zwischen dem Handelsplatz und dem Clearinghaus - nicht zuletzt aufgrund von Risikomanagement-Erwägungen charakterisiert sind, werden aufbrechen und eine grundlegende Neuordnung erfahren. Neue Handelsplätze und Clearinganbieter könnten in den Markt eintreten und den Wettbewerb 
verschärfen. Allerdings werden in diesem Falle Regulatoren und Aufsichtsbehörden gefordert sein, dass dies nicht zu einer Erhöhung der systemischen Risiken führt und die Funktionsweise der europäischen Derivatemärkte nicht beeinträchtigt wird.

\section{Literaturverzeichnis}

Bundesgesetzblatt (2013): “Gesetz zur Vermeidung von Gefahren und Missbräuchen im Hochfrequenzhandel (Hochfrequenzhandelsgesetz)". Jahrgang 2013 Teil I Nr. 23, Bonn.

Degryse, H./De Jong, F./Van Kervel, V. (2011): "The Impact of Dark Trading and Visible Fragmentation on Market Quality", TILEC Discussion Paper Nr. 2011-026.

http://papers.ssrn.com/sol3/papers.cfm?abstract_id=1815025 (Zugriff: 30.04.2014).

Europäische Kommission (2002a): “Vorschlag für eine Richtlinie über Wertpapierdienstleistungen und geregelte Märkte", 2002/0269 (COD).

Europäische Kommission (2002b): "Wertpapierdienstleistungen: vorgeschlagene neue Richtlinie soll Anleger schützen und EU-weite Tätigkeit von Wertpapierhäusern erleichtern", Pressemitteilung, IP/02/1706.

http://europa.eu/rapid/press-release_IP-02-1706_de.htm (Zugriff: 13.05.2014).

Europäische Kommission (2011): “Vorschlag für eine Richtlinie über Märkte für Finanzinstrumente zur Aufhebung der Richtlinie 2004/39/EG des Europäischen Parlaments und des Rates", 2011/0298 (COD).

Europäisches Parlament und Rat (2004): "Richtlinie über Märkte für Finanzinstrumente", 2004/39/EG (MiFID), in: Amtsblatt der Europäischen Union, L145/1 vom 30.4.2004.

Europäisches Parlament und Rat (2014a): „Richtlinie 2014/65/EU vom 15. Mai 2014 über Märkte für Finanzinstrumente sowie zur Änderung der Richtlinien 2002/92/EG und 2011/61/EU (MiFID II), in: Amtsblatt der Europäischen Union, L 173/349 vom 12.6.2014.

Europäisches Parlament und Rat (2014b): „Verordnung (EU) Nr. 600/2014 vom 15. Mai 2014 über Märkte für Finanzinstrumente und zur Änderung der Verordnung (EU) Nr. 648/2012 (MiFIR), in: Amtsblatt der Europäischen Union, L173/84 vom 12.6.2014.

European Securities and Markets Authority (2014a): MiFID Database. http://mifiddatabase.esma.europa.eu (Zugriff: 20.05.2014).

European Securities and Markets Authority (2014b): "Discussion Paper MiFIDII/MiFIR" http://www.esma.europa.eu/system/files/2014-548_discussion_paper_mifid-mifir.pdf (Zugriff: 13.06.2014).

European Securities and Markets Authority (2014c): "Consultation Paper MiFIDII/MiFIR" http://www.esma.europa.eu/system/files/2014-549_-_consultation_paper_mifid_ii_-_mifir.pdf (Zugriff: 13.06.2014).

Ferrarini, G. (2007): "Best Execution and Competition Between Trading Venues - MiFID's Likely Impact", in: Capital Markets Law Journal, Vol. 2, Nr. 4, S. 404-413.

Fidessa (2014): Fidessa index. http://fragmentation.fidessa.com/ (Zugriff: 13.05.2014). 
Gomber, P./Gsell, M./Lutat, M. (2011 a): "Competition among electronic markets and market quality", in: 14th Conference of the Swiss Society for Financial Market Research (SGF).

Gomber, P./Lutat, M./Pierron, A./Weber, M. C. (2011 b): "Shedding Light on the Dark - OTC Equities Trading in Europe", in: Journal of Trading, Vol. 6, Nr. 1, S. 74-86.

Gomber, P./Pujol, G./Wranik, A. (2012): "Best Execution Implementation and Broker Policies in Fragmented European Equity Markets", in: International Review of Business Research Papers, Vol. 8, Nr. 2, S. 144-162.

Gresse, C. (2012): "Effects of Lit and Dark Trading Venue Competition on Liquidity: The MiFID Experience", SSRN Working Paper.

http://papers.ssrn.com/sol3/papers.cfm?abstract_id=1918473 (Zugriff: 30.04.2014).

Hengelbrock, J./Theissen, E. (2009): "Fourteen at One Blow: The Market Entry of Turquoise", SSRN Working Paper.

http://papers.ssrn.com/sol3/papers.cfm?abstract_id=1570646 (Zugriff: 30.04.2014).

Oxera (2011): "Monitoring prices, costs and volumes of trading and post-trading services", http://www.oxera.com/Oxera/media/Oxera/downloads/reports/Oxera-report-on-trading-and-posttrading-May-2011.pdf?ext=.pdf (Zugriff: 13.05.2014).

Petrella, G. (2010): "MiFID, Reg NMS and Competition Across Trading Venues in Europe and United States", in: Journal of Financial Regulation and Compliance, Vol. 18, Nr. 3, S. 257-271.

Puaar, A. (2012): "MEPs seek to outlaw broker crossing networks", The TRADE News. http://thetradenews.com/news/Regions/Europe/MEPs_seek_to_outlaw_broker_crossing_networks. aspx (Zugriff: 14.05.2014).

Riordan, R./Storkenmaier, A./Wagener, M. (2011): "Do Multilateral Trading Facilities Contribute to Market Quality?", SSRN Working Paper.

http://papers.ssrn.com/sol3/papers.cfm?abstract_id=1852769 (Zugriff: 30.04.2014).

Soltani, B./Mai, H./Jerbi, M. (2011): "Transparency and Market Quality: An Analysis of the Effect of MiFID on Euronext (May 1, 2011). International Conference of the French Finance Association (AFFI), May 11-13, 2011. http://papers.ssrn.com/sol3/papers.cfm?abstract_id=1833605 (Zugriff: 30.04 . 2014).

Thomson Reuters (2014): „Monthly Market Share Reports“, http://thomsonreuters.com/monthlymarket-share-reports/ (Zugriff: 20.05.2014). 